\title{
The UNC-73/Trio RhoGEF-2 domain is required in separate isoforms for the regulation of pharynx pumping and normal neurotransmission in C. elegans
}

\author{
Robert Steven, ${ }^{1}$ Lijia Zhang, ${ }^{1}$ Joseph Culotti, ${ }^{1,2}$ and Tony Pawson ${ }^{1,2,3}$ \\ ${ }^{1}$ Samuel Lunenfeld Research Institute, Mount Sinai Hospital, Toronto, Ontario M5G 1X5, Canada; ${ }^{2}$ Department of Medical \\ Genetics and Microbiology, University of Toronto, Toronto, Ontario M5S 1A8, Canada
}

In both Caenorhabditis elegans and Drosophila, UNC-73/Trio functions in axon guidance by signaling through the Rac GTPase to regulate cytoskeletal rearrangements necessary for growth cone migrations. Here, we show that the complex $C$. elegans unc-73 gene encodes at least eight differentially expressed UNC-73 intracellular protein isoforms. Previously reported mutations affecting UNC-73 isoforms encoding the Rac-specific RhoGEF-1 domain cause uncoordinated movement, correlating with defects in axon guidance. Mutations in isoforms encoding the Rho-specific RhoGEF-2 domain, which we describe here, result in L1 stage larval lethality with no associated axon guidance defects. Isoform-specific rescue experiments reveal separate functions for the various RhoGEF-2-containing UNC-73 isoforms, which would not likely be discovered by conventional genetic screening. UNC-73 D1 and D2 appear to function redundantly in pharynx muscle to regulate the rate and strength of pharynx pumping, and in the HSN neurons and vulval muscles to control egg laying. Isoforms C1, C2, E, and F act redundantly within the nervous system to regulate the speed of locomotion. The multiple UNC-73 isoforms containing Rac- and Rho-specific RhoGEF domains therefore have distinct physiological functions. In addition to its previously identified role involving RhoGEF-1 in migrating cells and growth cones, our data indicate that UNC-73 signals through RhoGEF-2 to regulate pharynx and vulva musculature and to modulate synaptic neurotransmission.

[Keywords: Rho; synapse; behavior; splicing]

Supplemental material is available at http://www.genesdev.org.

Received March 31, 2005; revised version accepted July 6, 2005.

Members of the Rho subfamily of Ras-like monomeric GTPases, including Rho, Rac, and Cdc42, are involved in a broad range of functions, including gene transcription, cell cycle progression, cell polarity, and, most notably, regulation of the actin cytoskeleton and cell morphology (Burridge and Wennerberg 2004). Rho GTPases control the formation and elimination of actin-based structures such as stress fibers, lamellipodia, and filopodia and are important for cell and neuronal growth cone migrations.

Ras-like GTPases, including those of the Rho subfamily, typically function as molecular switches; they are active when bound to GTP and inactive when bound to GDP. In their active form, GTPases recruit downstream effector molecules, thereby engaging specific intracellular pathways. Three different types of molecules regulate GTPases as they cycle between active and inactive

${ }^{3}$ Corresponding author.

E-MAIL pawson@mshri.on.ca; FAX (416) 586-8869.

Article and publication are at http://www.genesdev.org/cgi/doi/10.1101/ gad.1319905. states. Rho GDP dissociation inhibitors associate with GDP-bound Rho family GTPases found in the cytosol and hold them in the inactive form by preventing the dissociation of GDP (Olofsson 1999). GTPase activating proteins increase intrinsic GTPase enzymatic activity, accelerating the conversion to the inactive GDP-bound form (Moon and Zheng 2003). Conversely, guanine nucleotide exchange factors (GEFs) stabilize the nucleotide free GTPase and since GTP is present in excess over GDP in the cytosol, they promote conversion to the GTP-bound active form (Rossman et al. 2005). Most molecules with RhoGEF activity contain a RhoGEF domain followed immediately by a pleckstrin homology $(\mathrm{PH})$ domain.

In pursuit of molecules that control cell and growth cone migrations we have previously characterized the Caenorhabditis elegans UNC-73 RhoGEF (Steven et al. 1998), which is related to mammalian Trio and Kalirin, and Drosophila Trio (Debant et al. 1996; Alam et al. 1997; Newsome et al. 2000). Mutations in unc-73 cause an uncoordinated movement phenotype, likely due to 
underlying axon patterning defects. unc-73 encodes proteins with several domains, including two tandem RhoGEF and PH domain combinations, a Sec14p motif, eight spectrin-like repeats, a variant SH3 domain, an immunoglobulin domain (Ig), and a fibronectin type III (FnIII) domain. The N-terminal UNC-73 RhoGEF-1 domain specifically activates the Rac family GTPases CED-10 and MIG-2 in vitro (Steven et al. 1998; Wu et al. 2002; Kubiseski et al. 2003), while the C-terminal RhoGEF-2 domain is specific to Rho (Spencer et al. 2001). Experiments in both C. elegans and Drosophila revealed that UNC-73/Trio function in axon guidance involves signaling through its RhoGEF-1 domain to the Rac GTPase and its downstream effectors, thereby regulating the cytoskeletal rearrangements necessary for growth cone migrations (Steven et al. 1998; Awasaki et al. 2000; Bateman et al. 2000; Liebl et al. 2000; Newsome et al. 2000; Lundquist et al. 2001; Wu et al. 2002; Ng and Luo 2004).

Here we report that unc-73 is a complex locus encoding at least eight RNA transcripts, several of which have not been described previously. Previously characterized unc-73 mutations cluster near the region encoding the RhoGEF-1 domain, and one has been shown to directly inactivate the Rac GEF activity of this domain. However, none of the new transcripts encoding the RhoGEF-2 domain are affected by any of the previously characterized mutations, including the canonical allele e936 or $\mathrm{gm} 40$, which was thought to represent a null allele because it introduces a stop codon near the start of the unc-73 coding sequence (Steven et al. 1998). Two unc-73 deletion alleles were isolated to examine the function of the transcripts encoding the RhoGEF-2 domain. One of these alleles was used in isoform-specific rescue experiments to determine the functions of the individual UNC-73 RhoGEF-2-containing isoforms. Two isoforms function redundantly in pharynx muscle to regulate pumping of the pharynx, and in the HSN neurons and vulval muscles to control egg laying. A different set of UNC-73 isoforms act redundantly within the nervous system to regulate the speed of locomotion. These data reveal that the unc-73 gene products have a remarkable molecular and biological complexity. Our genetic analysis, the nature of the phenotypes, and the fact that we do not observe axon patterning defects in the new alleles lead us to propose that in addition to its role in the growth cone, UNC-73 also functions in the process of neurotransmission through its RhoGEF-2 domain.

\section{Results}

\section{Structure of the unc-73 transcripts}

Two transcripts encoded by the unc-73 locus were previously identified (Steven et al. 1998) and named A and B (Fig. 1; Supplemental Material). A comparison of C. elegans and Caenorhabditis briggsae genomic DNA (Fig. 2) and further Northern blotting experiments (Fig. 1) indicated the presence of six additional unc-73 transcripts $(\mathrm{C} 1, \mathrm{C} 2, \mathrm{D} 1, \mathrm{D} 2, \mathrm{E}$, and F) whose structures were determined and confirmed by the analysis of cDNAs and/or

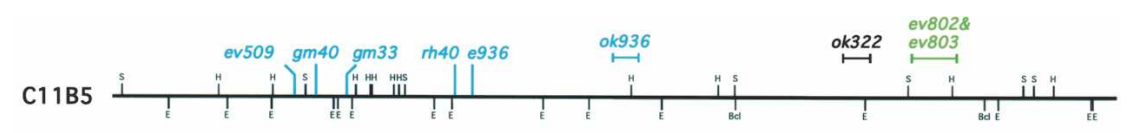

$\triangle \mathrm{C} 11 \mathrm{~B} 5$

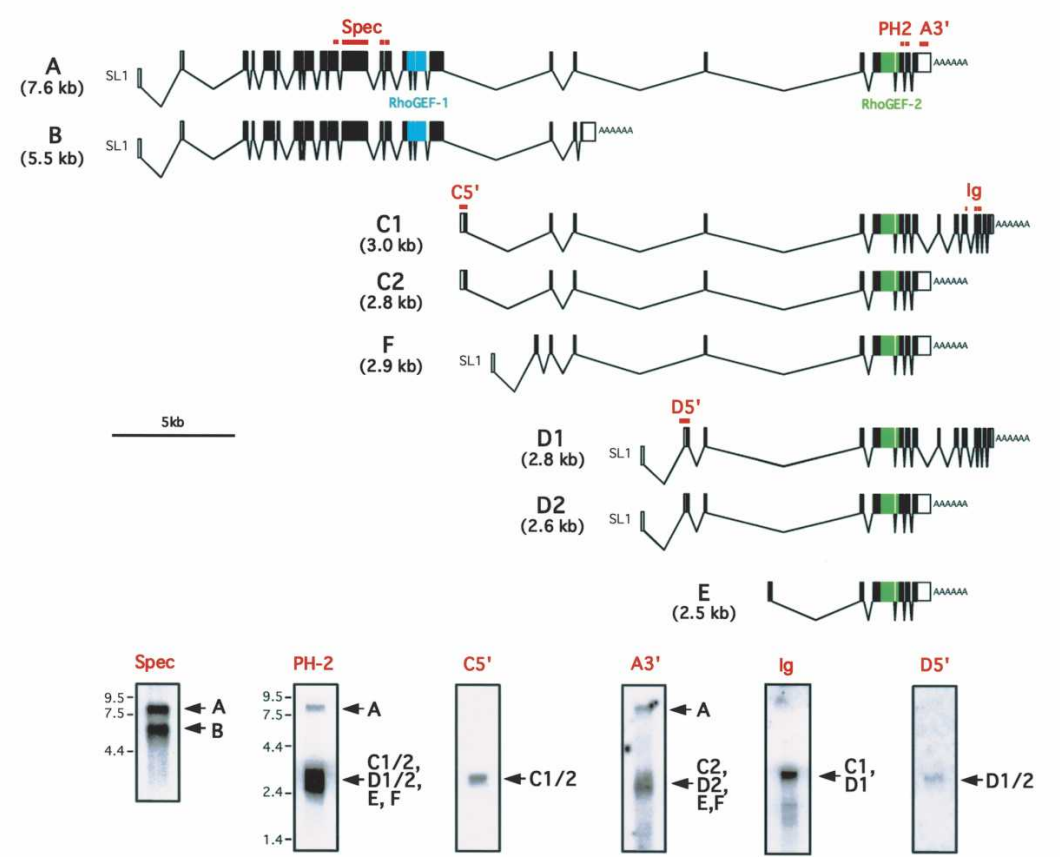

Figure 1. Structures of the predicted unc73 transcripts. The unc-73-containing cosmid, C11B5, and a deleted version, $\triangle \mathrm{C} 11 \mathrm{~B} 5$, are represented at the top of the figure. The locations of several identified mutations in the unc-73 gene are indicated on or above the cosmid. The mutations in blue result in an Unc phenotype, and those in green are associated with L1 lethality. Exons of the predicted unc-73 transcripts $(\mathrm{A}, \mathrm{B}, \mathrm{C} 1, \mathrm{C} 2$, D1, D2, E, and F) are shown to scale as filled (coding) or open (noncoding) boxes with the regions encoding the RhoGEF-1 and RhoGEF-2 domains in blue and green, respectively. See Figure 2 for exon numbering. SL1 is a trans-spliced $5^{\prime}$ leader sequence. The red lines above the transcripts represent the probes used for the accompanying Northern blots. The size markers drawn on the left side of the PH-2 blot also apply to the remaining unmarked blots. In some cases, the small differences in size between the six smaller transcripts do not allow the individual transcripts to be resolved on the blot and, as a result, multiple transcripts appear as one band on the Northern blot. Note that there is a splice variation at intron 25, represented by the cDNA yk1532c04, which is not shown on this figure but is described in the Supplemental Material. 
Steven et al.

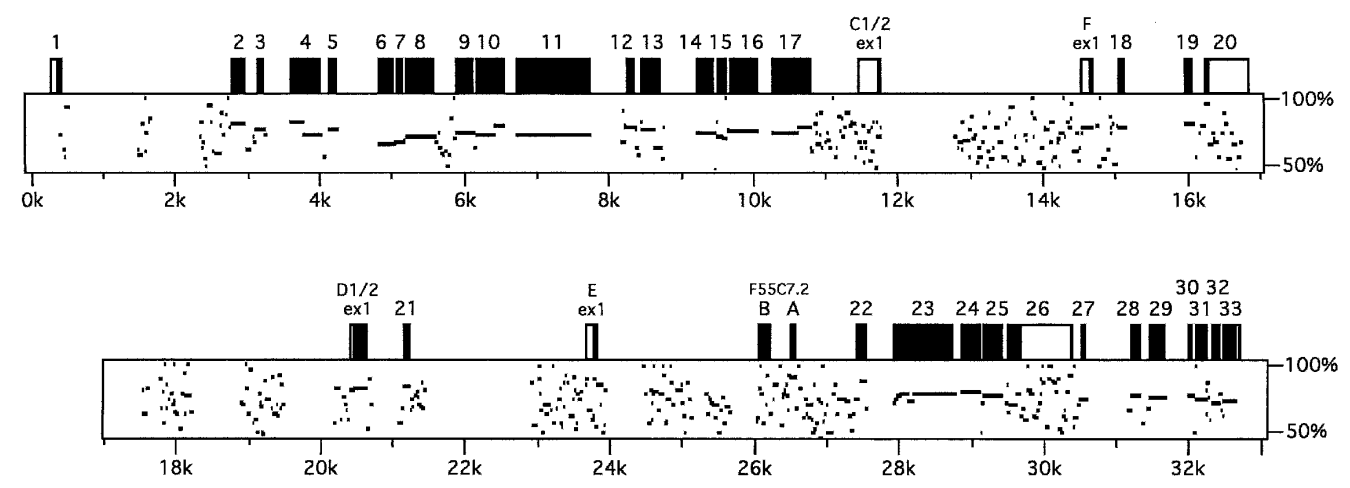

Figure 2. C. elegans versus C. briggsae percentage identity plot for the unc-73 region. The genomic sequences of $C$. elegans and $C$. briggsae are compared by plotting the percentage identities for short regions of unc-73 as horizontal lines on a graph using the Pipmaker algorithm at http://bio.cse.psu.edu/pipmaker. The horizontal axis represents the length of the unc-73 gene. The vertical axis indicates the level of identity between $50 \%$ and $100 \%$. The numbered boxes above the plot delineate the unc- 73 exons. Open boxes indicate untranslated regions. The plot indicates the high sequence conservation between the two genomes in the regions encoding the unc-73 exons. Possible promoter elements are indicated on the plot by the "clouds" of short sequence identity 5 " of the first exons of the different transcripts. The two exons (A and B) of the predicted gene F55C7.2 (Supplemental Material) are also indicated. The C. elegans DNA clones C09D1 and F55C7 and the C. briggsae clone CB032N22 (accession nos. AF003131, U80436, and AC087076, respectively/ were used for the comparison.

RT-PCR products (Supplementary Fig. S1; Supplementary Table S1). There is a large amount of sequence overlap between the eight predicted unc-73 transcripts. Virtually all of the unc-73 coding sequence is found in the combined A and C1 transcripts, with the remaining transcripts consisting of shorter variations of $\mathrm{A}$ and $\mathrm{C} 1$.

The protein isoforms encoded by the A, B, C1, C2, and $\mathrm{F}$ transcripts were immunoprecipitated and visualized on a Western blot using a polyclonal antibody raised to a C-terminal segment of isoform B (Fig. 3A; Steven et al. 1998). This segment is not found in the D1, D2, and E isoforms, so these isoforms were not visible on the blot. $\mathrm{A}$ and $\mathrm{B}$ migrated with the predicted mobilities based on their calculated molecular weights of 280 and $190 \mathrm{kDa}$, respectively, while $\mathrm{C} 1, \mathrm{C} 2$, and $\mathrm{F}$ migrated slightly slower than expected for their calculated molecular weights of 102,70 , and $74 \mathrm{kDa}$, respectively. C1 ran as a doublet, potentially due to post-translational modification. Identification of the particular UNC-73 isoforms on this Western blot of endogenous proteins was aided by an additional Western blot, where the individual isoforms were distinguished by a GFP tag (Fig. 3B).

\section{Deletion of the unc-73 RhoGEF-2 region is lethal}

All of the previously reported mutations in unc-73 result in a $100 \%$ penetrant uncoordinated movement (Unc) phenotype reflecting errors in axon guidance during the development of the nervous system (Steven et al. 1998). None of these unc-73 mutations are predicted to affect the production or coding potential of any of the six smaller transcripts (C1, C2, D1, D2, E, or F) identified in this paper (Fig. 1). This includes the strong allele gm40, which was previously anticipated to be a null allele. Indeed, a Western blot of unc-73(e936) protein lysate revealed wild-type levels of $\mathrm{C} 1, \mathrm{C} 2$, and $\mathrm{F}$ but very low levels of A and B (Fig. 3).
To examine the function of the six smaller unc-73 transcripts that encode the RhoGEF-2 domain, we isolated two deletion mutant alleles, ev802 and ev803, which eliminate the unc-73 RhoGEF-2 genomic region (Fig. 1; Supplementary Table S2). ev802 and ev803 mutants arrested development at the L1 stage but continued to survive for several days. They left normal sinusoidal tracks in the bacterial lawn and did not display uncoordinated movement defects typical of previously reported unc-73 mutants. Visualization of the nervous system in ev802 mutants with a nervous system-specific GFP reporter (F25B3.3p::GFP) failed to detect any defects in axon patterning (data not shown).

Although the ev802 strain was backcrossed five times to wild type (N2), we nonetheless sought additional evidence that the lethal phenotype was due to the deletion in unc-73 and not another tightly linked mutation. The cosmid C11B5 (Fig. 1) rescued ev802 lethality, and as previously reported, C11B5 also rescued the Unc phenotype of all of the unc-73 alleles tested (Steven et al. 1998). A 5' deleted C11B5 cosmid ( $\Delta \mathrm{C} 11 \mathrm{~B} 5)$ (Fig. 1) also rescued ev802 but not the Unc alleles $e 936$, gm40, or $r h 40$. Conversely, the unc-73 minigene, pNW217, which encodes only the B transcript and can rescue the unc-73 Unc alleles (Steven et al. 1998), did not rescue ev802 lethality.

These rescue experiments not only suggest that the deletion encompassing the RhoGEF-2 sequence in unc73 is the cause of the lethality in ev802 mutants, they also reveal that mutations in transcript A can be compensated by the presence of wild-type copies of all the remaining transcripts $B$ through $F$. That is to say, regardless of the mutations in transcript A, including a severe truncation in the case of $g m 40$, an Unc mutant animal requires only wild-type transcripts $\mathrm{B}$ through $\mathrm{F}$ for rescue to wild-type function. In support of this contention, we have observed intragenic complementation between the Unc alleles and the ev802 allele of unc-73; for example, 
A

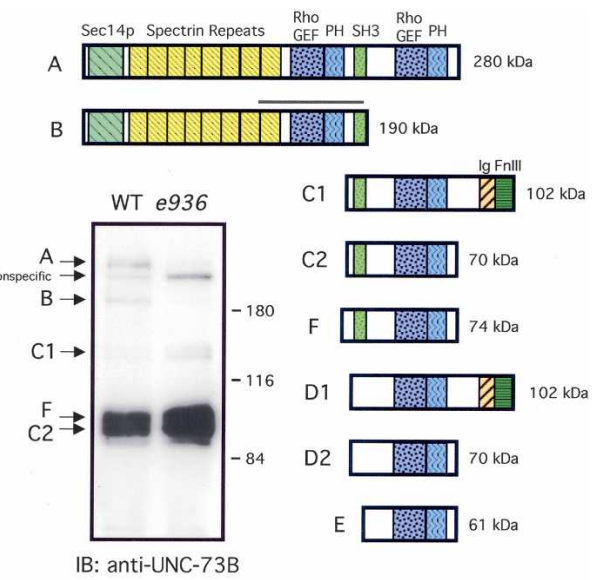

B

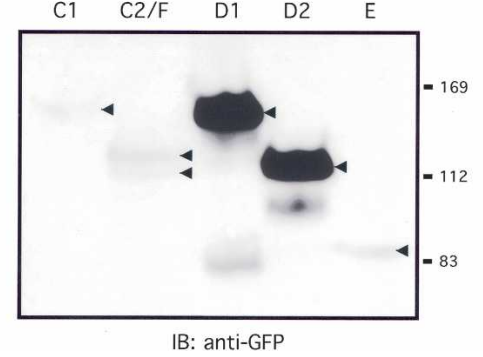

Figure 3. Structures of the predicted UNC-73 protein isoforms. (A) The predicted UNC-73 isoforms are drawn to scale. An antibody raised to the portion of UNC-73 indicated by the red line was used to immunoprecipitate UNC-73 from soluble lysates of mixed stage wild-type and unc-73(e936) animals. The immunoprecipitated proteins were separated and blotted, and then probed with the same UNC-73 antibody. The A, B, C1, C2, and $\mathrm{F}$ bands on the Western are competed away by the UNC-73 antigen, but the indicated nonspecific band is not (data not shown). The $\mathrm{C} 1$ isoform appears to run as a doublet. (B) A Western blot of C. elegans lysates from ev802 strains containing constructs encoding the indicated UNC-73 isoforms fused to GFP. The strains containing C1, C2/F, and E fused to GFP also have a construct encoding D1 (not fused to GFP) to rescue ev802 lethality. The blot is probed with a polyclonal antibody to GFP.

ev802/gm40, ev802/rh40, and ev802/ok936 animals have a wild-type phenotype (data not shown). It thus appears that there is no unique role for the unc-73 A transcript, and the $\mathrm{B}$ transcript has functions separable from the six smaller unc-73 transcripts, C1 through F. This observation also raises the possibility that one of the early larval lethal genes, let-373, let-510, or let-511, which map close to unc-73, may be allelic to unc-73(ev802).

\section{unc-73(ev802) mutants display abnormal pharynx pumping behavior}

Developmental arrest at the L1 stage can occur as a result of starvation; therefore, functional deficits in the pharynx, where the D1 and D2 isoforms are expressed (Fig. 4), may explain the developmental arrest of ev802 mutants. We measured the pharynx pumping rate of sev- eral L1 progeny from unc-73(ev802)/unc-11 dpy-5 parents. The ev802 pumping rate was reduced to $91 \pm 29$ pumps/min compared with $208 \pm 22$ pumps/min for unc73(ev802)/unc-11 dpy-5 siblings (Fig. 5A). The quality of pharynx muscle contractions was also different in ev802 animals. Although the corpus appeared to contract normally, but at a slower rate, and isthmus peristalsis was still evident, the muscles in the terminal bulb contracted only weakly, more like a twitch compared with the strong contraction seen in control unc-73(ev802)/unc-11 dpy-5 or N2 animals. Very few bacteria entered the digestive tract of ev802 animals, and the lumen of the gut remained large and mostly empty in these animals. ev802 animals fed on a mixture of bacteria and fluorescent beads had very few beads in their gut compared with wild-type animals whose digestive tracts filled with beads (data not shown). The weak pharynx contraction phenotype is likely an important aspect of the lethal phenotype since a reduced pumping rate cannot alone explain the ev802 lethal phenotype as viable unc-11 dpy-5 siblings had a similarly reduced rate of $96 \pm 17$ pumps/ min (Fig. 5A). Mutations in unc-11, which encodes the clathrin adaptor protein AP180, were previously reported to cause a reduced pharyngeal pumping rate (Nonet et al. 1999).

The D1 and D2 isoforms rescue unc-73(ev802) lethality, but the rescued animals display a lethargic phenotype

The functions of the multiple unc-73 isoforms were examined by testing the abilities of individual isoform-specific constructs (Materials and Methods) to rescue the lethality of ev802 mutants. Tested individually, both the $\mathrm{D} 1$ and D2 isoforms, which are strongly expressed in the pharynx (Fig. 4), rescued ev802 lethality. [D1] ev802 and [D2] ev802 animals had wild-type pharynx pumping rates (Fig. 5B), and the strength of pharynx contractions was normal. Rescue was not obtained by individual injection of $\mathrm{C} 1, \mathrm{C} 2 / \mathrm{F}$, or $\mathrm{E} / \mathrm{C} 2$ and $\mathrm{F}$ are encoded by a single construct; see Materials and Methods), which are not expressed in pharyngeal muscle (Fig. 4).

Although larval lethality was rescued, [D1] ev802 and [D2] ev802 hermaphrodite animals had an intriguing lethargic movement defect. The movement of these animals was smooth and coordinated with normal tracks left in the bacterial lawn, but their rate of movement was very slow. Their speed increased in the absence of bacteria, but it was still about one-third the rate of wild type (Fig. 6A). ev802 L1 animals without D1 also displayed the lethargic movement phenotype (Fig. 6B). Surprisingly, [D1] ev802 males were not lethargic, and brief periods of normal movement on bacteria could be induced in [D1] ev802 hermaphrodites by prodding with a worm pick (data not shown).

The preceding experiments used individual isoform microinjections. We next tested if different combinations of unc-73 isoforms rescued [D1] ev802 or [D2] ev802 lethargy (Table 1). All injected combinations included either D1 or D2 to rescue ev802 lethality. Al- 


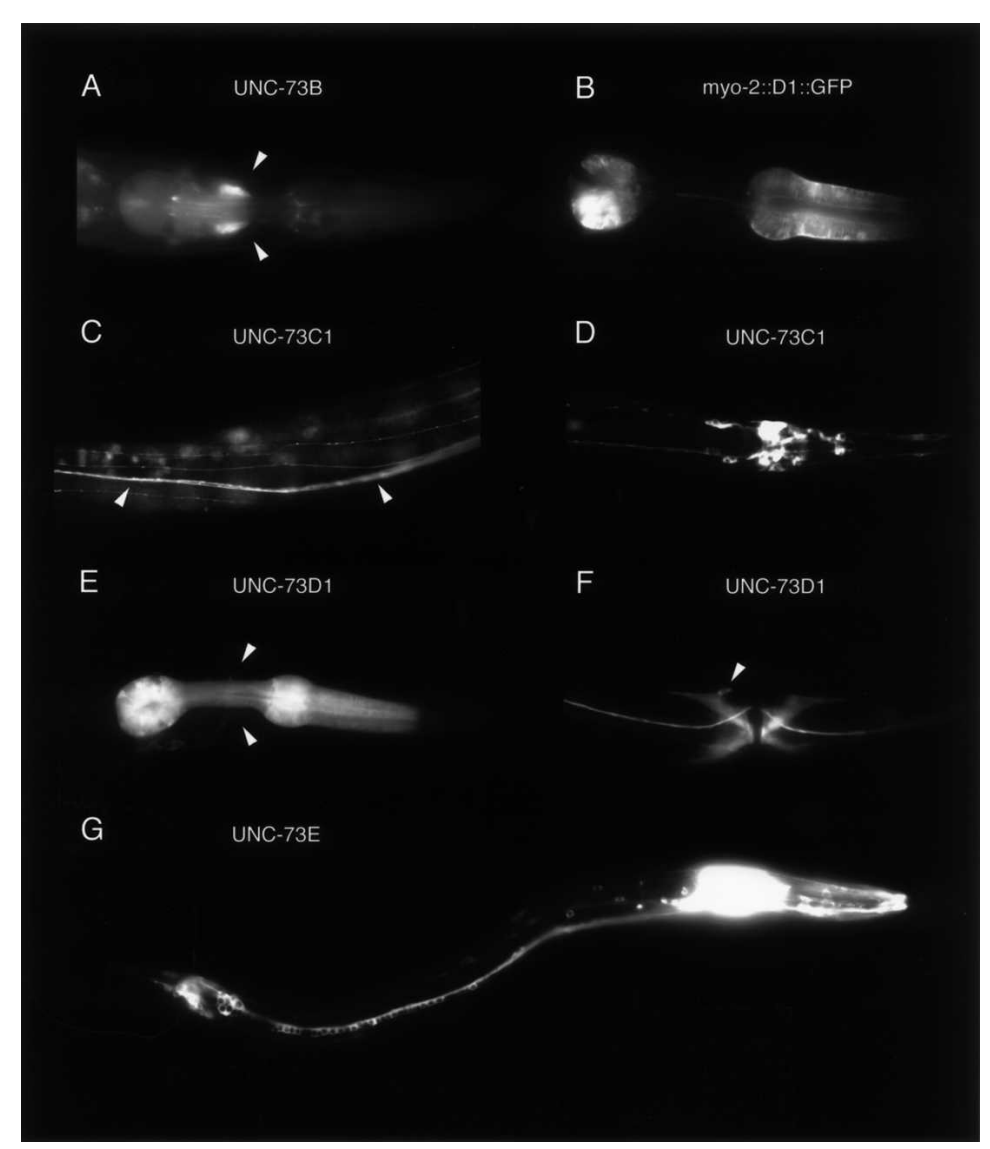

Figure 4. Expression patterns of the unc-73 isoforms. The patterns of expression of the unc-73 isoforms B through $\mathrm{F}$ were determined using constructs that each specifically encode an individual unc-73 isoform tagged with GFP at the $\mathrm{C}$ terminus. The constructs were injected into wild-type animals, and the larvae and adults of the resulting stable lines were examined. All animals are oriented with the posterior to the left and anterior to the right. (A) UNC-73 B is strongly expressed in the axons of neurons. A cross-sectional side view of a latelarval stage animal reveals the nerve ring as two bright patches of fluorescence around the pharynx (arrowheads). (B) Expression of D1 driven by the myo-2 promoter in [myo-2p::D1::GFP] ev802/unc-11 dpy-5 animals is limited to the muscles of the pharynx. The $\mathrm{C} 1$ isoform is expressed in a limited set of neurons in the midbody of the animal $(C)$ and the head $(D)$. Arrowheads indicate the ventral cord. Also visible in the head are the neuronal support socket and/or sheath cells. The C2/F isoform has the same pattern of expression as C1. (E) D1 expression in the muscles of the pharynx and two neurons in the head. A few unidentified axons are faintly visible in a cross-section of the nerve ring (arrowheads). (F) The processes of D1-expressing neurons are visible in the ventral cord. The muscles of the vulva seen in the shape of an " $X$ ", and the HSNR cell body (arrowhead) also express D1. The D2 isoform has the same pattern of expression as D1. (G) UNC-73 E is expressed in the axons and cell bodies of most if not all neurons and the excretory canals. The bright region in the head is a result of expression in axons of the nerve ring and the cell bodies of neurons in the ganglia surrounding the nerve ring. though a mixture of $\mathrm{C} 1, \mathrm{C} 2 / \mathrm{F}$, and $\mathrm{E}$ rescued the lethargic movement defect, injections of fewer isoforms also resulted in rescue, indicating that some redundancy exists between $\mathrm{C} 1, \mathrm{C} 2 / \mathrm{F}$, and $\mathrm{E}$, all of which are expressed in the nervous system (Table 1; Figs. 4, 6A). Therefore, it appears that the minimal requirement for complete rescue of ev802 is a combination of either D1 or D2 to rescue the lethality and either $\mathrm{C} 1, \mathrm{C} 2 / \mathrm{F}$, or $\mathrm{E}$ to rescue the lethargy.

\section{The UNC-73 isoforms are differentially expressed}

The expression patterns of unc-73 isoforms B through $\mathrm{F}$ were determined in larvae and adults using constructs encoding the individual isoforms tagged with GFP (Materials and Methods; Fig. 4). All of the GFP-tagged constructs retained their ability to rescue mutant phenotypes except for those encoding the $\mathrm{C} 1$ and $\mathrm{C} 2 / \mathrm{F}$ isoforms. Isoform $\mathrm{B}$ was expressed most strongly in the axons of neurons, particularly in the nerve ring (Fig. 4A) and the ventral nerve cord. This pattern of expression was very similar to the staining pattern observed with UNC-73 B antibodies (Steven et al. 1998). UNC-73 B was also found more sporadically and at a lower level in anal depressor muscle, distal tip, $\mathrm{P}$, seam, and developing vulva cells (data not shown). Although $\mathrm{C} 1$ and C2/F were also expressed in axons, their expression was restricted to fewer neurons (Fig. 4C). Many process bundles within the body of the animal and neurons within the pharynx were positive for $\mathrm{C} 1$ and $\mathrm{C} 2 / \mathrm{F}$, but few axons were visible in the nerve ring (Fig. 4D). Along with the neuronal expression, $\mathrm{C} 1$ and $\mathrm{C} 2 / \mathrm{F}$ were also expressed in neuronassociated socket and/or sheath cells (Fig. 4D) and the neuroendocrine uv1 cells (data not shown). A low level of expression was sporadically observed in body wall muscles (data not shown).

In contrast, D1 and D2 were very strongly expressed in the muscles of the pharynx and vulva and in both the cell bodies and axons of a very restricted set of neurons (Fig. $4 \mathrm{E}, \mathrm{F}$; data not shown). These included the hermaphrodite-specific neurons (HSNs), about four unidentified neurons with cell bodies in either the ventral or retrovesicular ganglion near the terminal bulb of the pharynx, and about six neurons with cell bodies in the lumbar ganglion in the tail, including PVQL and PVQR, as distinguished by their axonal patterning. A small number of axons were present in the nerve ring and the ventral cord. The lateral processes of ALNL and ALNR were also visible. D1 and D2 also exhibited sporadic and weak fluorescence in body wall and intestinal muscles (data not shown). Isoform E was strongly expressed in the nervous system and was detected in the cell bodies and axons of most, if not all neurons, including those in the pharynx, and at least some of the neuron-associated sheath and/or socket cells (Fig. 4G). Isoform E was also observed in the excretory canals and the somatic gonad, 
A

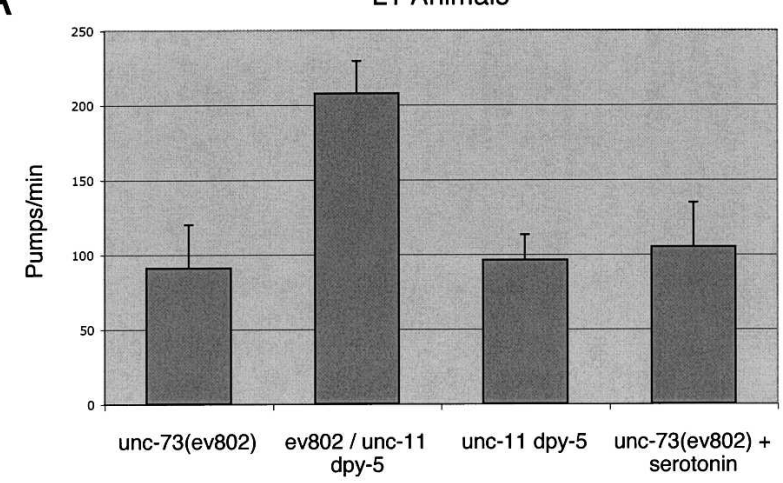

B

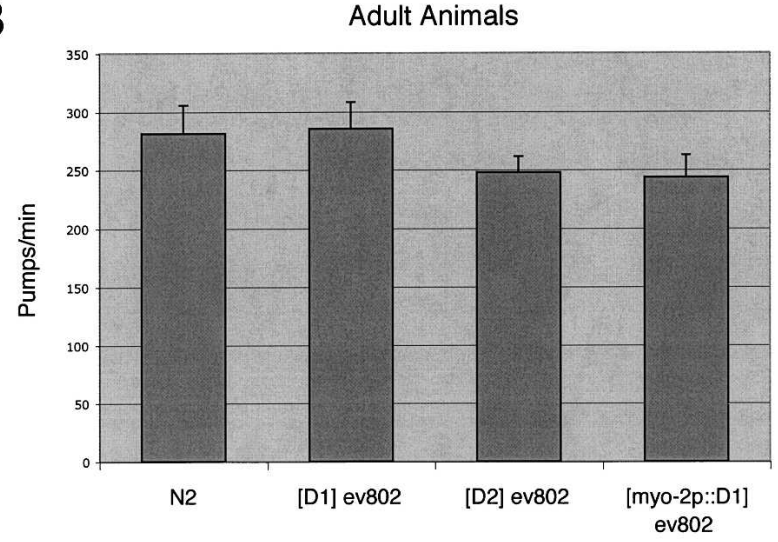

Figure 5. The pharynx pumping rate is reduced in unc73(ev802) animals. Plots of the pharynx pumping rate in pumps per minute. $(A)$ Rates for L1 stage ev802 $(n=14)$, unc-73(ev802)/ unc-11 dpy-5 $(n=30)$, and unc-11 dpy-5 $(n=14)$ animals. The pumping rate is reduced in ev802 and unc-11 homozygotes. (B) Rates for young-adult N2 (wild type) $(n=20),[D 1]$ ev802 $(n=20),[\mathrm{D} 2]$ ev802 $(n=22)$, and [myo-2p::D1] ev802 $(n=22)$ animals. The reduced pumping rate in ev802 animals is rescued to wild-type levels with the presence of the D1, D2 or myo$2 \mathrm{p}:: \mathrm{D} 1$ isoform transgenes.

including the spermatheca, gonadal sheath, and distal tip cells (data not shown).

Restricted expression of D1 in pharyngeal muscle is sufficient for rescue of unc-73(ev802) lethality

The importance of the pharynx for viability and expression of D1 and D2 in the pharyngeal muscles of wildtype animals (Fig. 4E) suggested that the lethality associated with ev802 and ev803 animals may result solely from the loss of UNC-73 D1 and D2 in the pharynx. D1 and D2, however, are also expressed in a small subset of neurons, which may connect directly or indirectly to the pharynx to influence its pumping action. To test the relevance of D1 pharyngeal expression, we used the myo-2 promoter to limit expression of D1 to muscles of the pharynx (Fig. 4B). [myo-2p::D1] ev802 animals developed normally with strong pharyngeal muscle contractions and wild-type rates of pumping (Fig. 5B). [myo2p::D1] ev802 animals were also lethargic (Fig. 6A).
These results indicate that D1 plays a role in pharynx muscle function since there was complete rescue of ev802 L1 arrest. Consistent with D1 functioning in the pharynx muscle, neither the rate nor quality of ev802 pumping was increased in the presence of serotonin (10 $\mathrm{mg} / \mathrm{mL}$ ), a stimulant of pharynx pumping (Fig. 4A; Horvitz et al. 1982). Complete rescue of ev802 to wild-type rates of movement was obtained by the combined injection of myo-2p::D1 and either C1 or E (Fig. 6A).

\section{UNC-73 D1 is required for egg laying}

Although D1 expression outside the pharynx was not required to rescue ev802 lethality, expression within at least a subset of these cells, most likely the HSN neurons and/or the vulval muscles, was required for the process of egg laying. [myo-2p::D1] ev802 animals, which lacked D1 expression in the vulval muscles and HSNs (data not shown), had an egg-laying defect (Egl). These animals displayed a "bag of worms" phenotype in which few eggs were laid and instead most hatched inside the animal (Fig. 7). The HSNs innervate the vulval muscles, and both are required to control the opening of the vulva to allow eggs to be laid (Trent et al. 1983). Expression of the E isoform in the HSNs of [myo-2p::D1; E] ev802 animals most likely accounted for the partial rescue of the Egl phenotype in these animals (Fig. 7).

\section{Differential requirements for the RhoGEF-2 domain}

The rh40 missense mutation in the unc-73 RhoGEF-1 domain revealed the importance of RhoGEF-1 catalytic activity to UNC-73 function (Steven et al. 1998). To examine the role of the RhoGEF-2 domain in the various UNC-73 isoforms, we separately introduced two different mutations into the D1 and C1 RhoGEF-2 domains.

The first mutation, C1984Y, which is equivalent to an alteration identified in unc-73(je3), is predicted to disrupt the conformation of the RhoGEF-2 domain (Supplemental Material). UNC-73 D1 and C1 isoforms containing the C1984Y mutation lost their biological activity: (C1984Y)D1 failed to rescue ev802 lethality, and (C1984Y)C1 failed to rescue the lethargic phenotype of [D1] ev802 animals (Fig. 6A). GFP-tagged versions of (C1984Y)D1 and (C1984Y)C1 displayed similar levels of fluorescence compared with wild-type D1::GFP and $\mathrm{C} 1:: \mathrm{GFP}$, indicating the mutations did not affect the stability or expression of the isoforms, although for unknown reasons, larger more extended CAN neuron cell bodies were occasionally observed upon expression of (C1984Y)C1 (data not shown). This experiment reveals the importance of the RhoGEF-2 domain to the function of both $\mathrm{C} 1$ and D1. It also demonstrates that the C1984Y mutation is the probable cause of unc-73(je3) lethality.

We also introduced a second RhoGEF-2 mutation, Q1944A, that is equivalent to a Q1368A substitution in the RhoGEF-1 domain of the UNC-73 mammalian homolog Trio. This mutation severely reduces Trio RhoGEF-1 catalytic activity in vitro (Liu et al. 1998). Interestingly, this mutation in UNC-73 D1 had no effect on its ability to rescue ev802 lethality, while the same 
Steven et al.

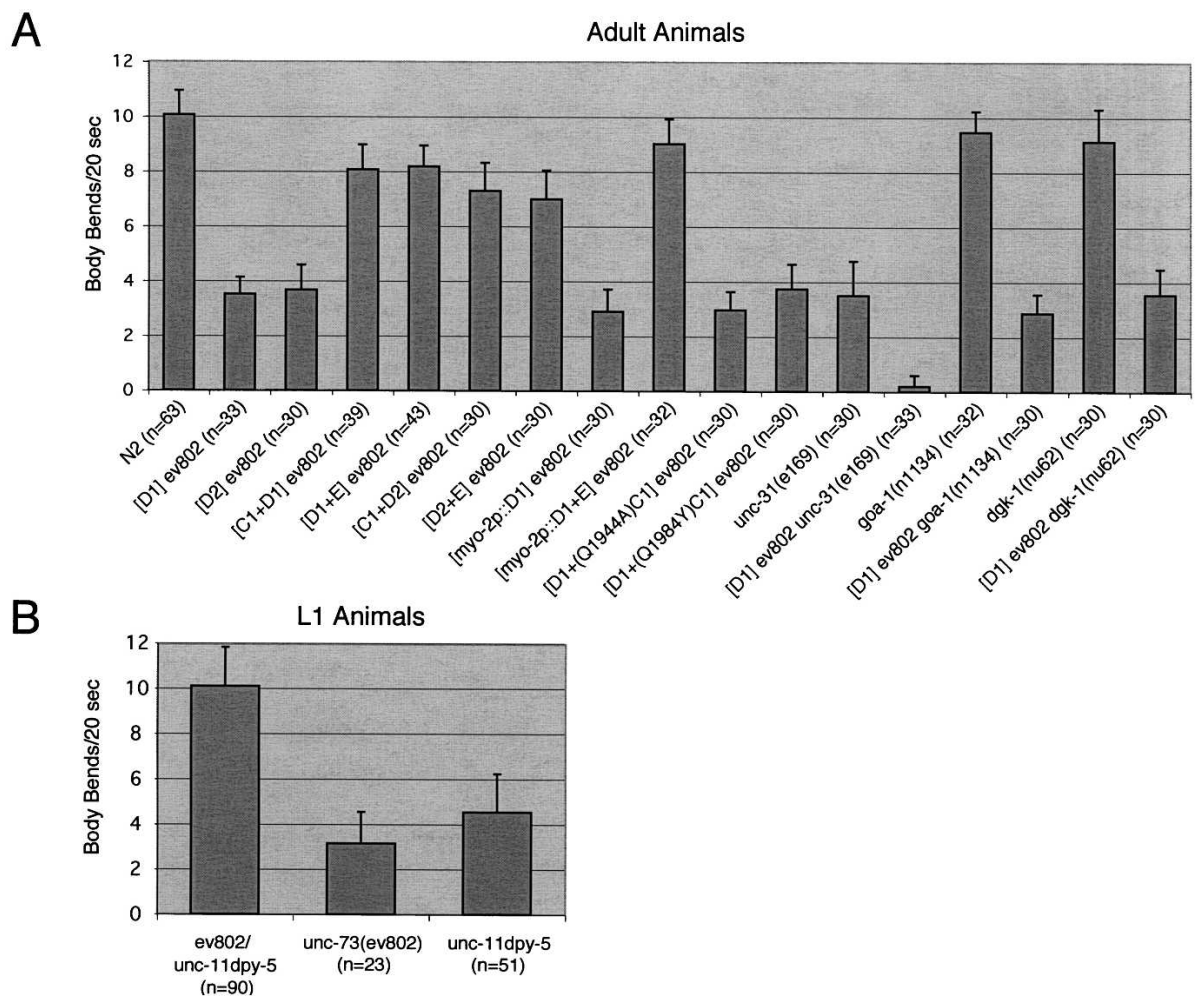

Figure 6. The rate of movement is reduced in unc-73(ev802) animals. The rates of movement for various strains are measured as body bends per 20 -sec interval as nematodes travel uninterrupted on agar plates free of bacteria. The movement rates of young adults $(A)$ and L1 stage animals $(B)$ are shown. The lethargy of ev802, [D1] ev802, [D2] ev802, and [myo-2p::D1] ev802 animals is rescued to wild-type levels with the presence of either the $\mathrm{C} 1$ or E isoform transgenes. $\mathrm{C} 1$ loses the ability to rescue the lethargy if it contains a mutation in the RhoGEF-2 domain.

mutation in $\mathrm{C} 1$ blocked the ability of $\mathrm{C} 1$ to rescue the [D1] ev802 lethargic phenotype (Fig. 6A). Although (Q1944A)D1 rescued ev802 lethality, [(Q1944A)D1] ev802 animals had an egg-laying defect (Fig. 7). This suggests that the Q1944A mutation did influence D1 function in either the HSNs or vulval muscles, which are important for egg laying, but it did not affect D1 function in the pharynx, where it is required to rescue ev802 lethality. (Q1944A)C1::GFP and (Q1944A)D1::GFP were expressed at the same level and in the same cells compared with $\mathrm{C} 1:: \mathrm{GFP}$ and D1::GFP (data not shown) indicating that Q1944A does not influence isoform stability or expression. These experiments identify a differential requirement for RhoGEF-2 activity in D1 expressing cells versus cells expressing $\mathrm{C} 1$. The fact that (Q1944A)D1 rescued ev802 lethality, whereas (C1984Y)D1 did not, suggests that the Q1944A substitution does not entirely ablate RhoGEF-2 function. These data, in turn, indicate that there may be a reduced requirement for RhoGEF-2 activity in pharynx muscle versus the requirement in cells of the nervous system and possibly the vulval muscles.

\section{UNC-73 likely influences neurotransmission}

Aside from their lethargy, [D1] ev802 hermaphrodites moved in an otherwise coordinated manner, and indeed, a wild-type rate of movement could be briefly induced by mechanical stimulation. UNC-73 C1, C2/F, and $\mathrm{E}$, which were all able to rescue the lethargic phenotype, have expression patterns that only overlap in the nervous system. These observations suggested that [D1] ev802 animals have defects in the nervous system affecting the regulation of locomotory speed. Since we did not find any neuronal patterning defects in [D1] ev802 animals, it is possible that $\mathrm{C} 1, \mathrm{C} 2 / \mathrm{F}$, and $\mathrm{E}$ are required for proper communication between neurons at the level of the synapse.

Most synapse gene mutants are resistant to the acetylcholinesterase inhibitor aldicarb (Miller et al. 1996), however, [D1] ev802 animals were not aldicarb resistant. One hundred percent of wild-type $(n=52)$ and [D1] ev802 $(n=116)$ animals placed for $4 \mathrm{~h}$ on plates containing 0.8 $\mathrm{mM}$ aldicarb were paralyzed and did not move upon mechanical stimulation. Eighteen percent of $s n b-1(m d 247)$ positive control animals $(n=55)$ were paralyzed under the same conditions. We also examined [D1] ev802 synapse structure using the synaptic vesicle markers unc25p::snb-1::gfp (juIs1) and sek-1p::snb-1::gfp expressed in the GABAergic system and an unidentified subset of neurons, respectively, and ida-1::gfp a dense core vesicle marker also expressed in a subset of neurons (Hallam and Jin 1998; Zahn et al. 2001). No difference in expression of the markers was discerned between [D1] ev802 and con- 
Table 1. The UNC-73 C1, C2/F, or E isoform can rescue the lethargic movement phenotype

\begin{tabular}{|c|c|c|c|c|c|c|c|c|}
\hline \multirow[b]{2}{*}{ UNC-73 Isoform } & \multicolumn{8}{|c|}{ Injected Construct Groups } \\
\hline & 1 & 2 & 3 & 4 & 5 & 6 & 7 & 8 \\
\hline 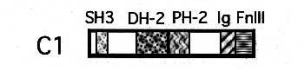 & + & + & + & & & & + & \\
\hline C2/F 圈 B & + & & & & + & & & \\
\hline 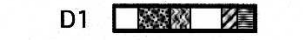 & + & + & + & + & + & + & & \\
\hline 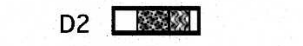 & + & & & & & + & + & + \\
\hline 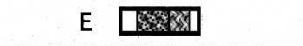 & + & + & & + & & & & + \\
\hline Rescue lethargic phenotype? & $\mathrm{Y}$ & Y & $\mathrm{Y}$ & $\mathrm{Y}$ & $\mathrm{Y}$ & $\mathrm{N}$ & $Y$ & $\mathrm{Y}$ \\
\hline
\end{tabular}

Different combinations of isoform-specific constructs were tested for their ability to rescue the lethargic movement phenotype of [D1] ev802 or [D2] ev802 animals. The lethality was rescued by the D1- or D2-specific constructs. All combinations, except for group number 6, gave rise to a wild-type movement phenotype in an ev802 background. Individually, either of the $\mathrm{C} 1, \mathrm{C} 2 / \mathrm{F}$, or $\mathrm{E}$ isoforms was capable of rescuing the lethargic movement phenotype.

trol [D1] ev802/unc-11 dpy-5 animals carrying these markers (data not shown).

Nematodes with defective mecanosensory neurons have a sluggish phenotype (Chalfie and Sulston 1981), but [D1] ev802 animals are sensitive to fine touch; therefore, their lethargy is not likely due to the inactivity of mecanosensory neurons. Mutations in the C. elegans genes, $r b f-1$ rabphilin, and unc-31 (known in vertebrates as CAPS for $\mathrm{Ca}^{2+}$-dependent activator protein for secretion) result in lethargic movement phenotypes that are similar yet less and more severe, respectively, than that of [D1] ev802 animals. The lethargy of $r b f-1$ animals is also overcome by mechanical stimulation and rabphilin mutants are, like [D1] ev802 animals, not resistant to aldicarb (Staunton et al. 2001). Genetic evidence in $C$. elegans points to a role for rabphilin in modifying the function of synaptic SNARE proteins (Staunton et al. 2001). unc-31 hermaphrodites are extremely sluggish on bacteria, less responsive to prodding than are [D1] ev802 animals, and are resistant to aldicarb (Avery et al. 1993; Miller et al. 1996). Like [D1] ev802 animals, however, unc-31 mutants move faster in the absence of bacteria, and on bacteria, unc-31 males move much faster than hermaphrodites (Fig. 6A; Avery et al. 1993).

Based on the potential for UNC-73 isoforms to play a role at the synapse, we searched for evidence of synergistic genetic interactions similar to those found for $r b f-1$ and known synapse function genes. Double mutants were constructed with [D1] ev802 and the genes rab-3(js49), rbf-1(js232) rabphilin, unc-10(md1117) RIM, snb-1(md247) synaptobrevin, snt-1(md290) synaptotagmin, unc-64(e246) syntaxin, unc-13(e51), and unc18(e81). Synergistic genetic interactions were not observed. In all cases, the defects in motility observed in a particular double mutant were no more or less severe than the defects observed in either of the two mutants in isolation (data not shown). A genetic interaction was ob- served, however, between [D1] ev802 and unc-31. The lethargy of [D1] ev802; unc-31(e169) animals off bacteria was much more severe compared with the movement of each single mutant (Fig. 6A), indicating that unc-31 and unc-73 probably act in parallel pathways.

Exogenous application of serotonin or dopamine decreases C. elegans motility, and both neurotransmitters are required for separate slowing responses in the presence of bacteria (Horvitz et al. 1982; Schafer and Kenyon 1995; Sawin et al. 2000). The heterotrimeric G protein subunit, $\mathrm{G}_{\mathrm{o}} \alpha$ (GOA-1), and diacylglycerol kinase (DGK1) signal downstream of serotonin and goa-1 and $d g k-1$ loss-of-function mutants are hyperactive (Segalat et al. 1995; Nurrish et al. 1999), while overexpression of goa-1 causes lethargic movement (Mendel et al. 1995). Also intriguing, considering UNC-73 RhoGEF-2 activity, is the fact that Rho physically associates with and inactivates DGK in mammalian cells (Houssa et al. 1999). These observations suggested that [D1] ev802 animals might have enhanced serotonin or dopamine signaling. However, mutations in bas-1 or tph-1, which encode enzymes required for serotonin and/or dopamine synthesis (Loer and Kenyon 1993; Sawin et al. 2000; Sze et al. 2000), and loss-of-function mutations in goa-1 or dgk-1 did not suppress [D1] ev802 lethargy (Fig. 6A; data not shown). The finding that [D1] ev802 lethargy is epistatic to goa-1 and $d g k-1$ hyperactivity indicates that UNC-73 $\mathrm{C} 1, \mathrm{C} 2 / \mathrm{F}$, and $\mathrm{E}$ function downstream of or in parallel to GOA-1 and DGK-1 signaling.

\section{Discussion}

The number of RhoGEF and RhoGAP regulators of Rho GTPases significantly exceeds the number of Rho family

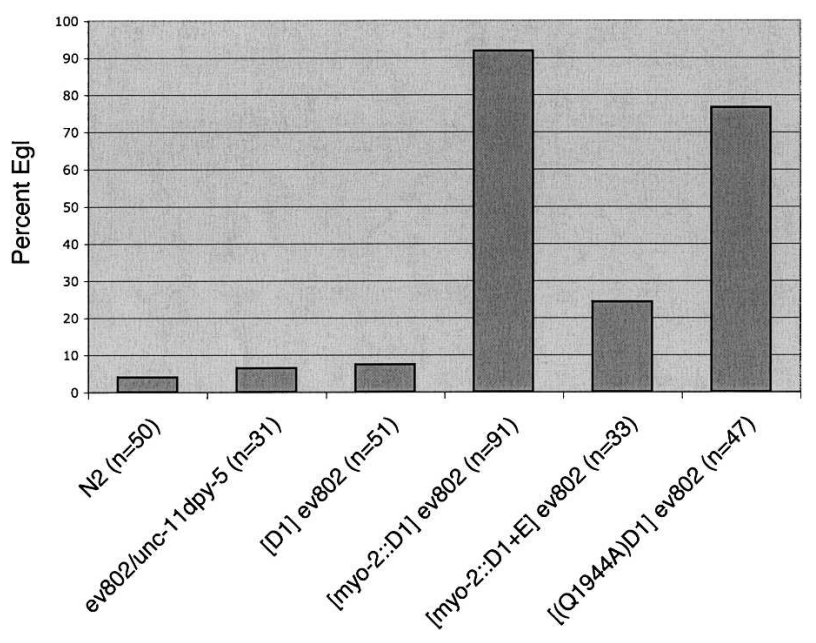

Figure 7. The UNC-73 D1 isoform is required for normal egg laying. The percentage of animals with an egg-laying defect (Egl) is plotted for each strain. An animal is defined as Egl if one or more of its eggs hatch inside its body within a period of $3 \mathrm{~d}$ of growth from the L4 stage. [myo-2p::D1] ev802 animals have a severe Egl phenotype. This defect is partially rescued by the addition of UNC-73 E. A Q1944A mutation in the D1 RhoGEF domain also results in an Egl phenotype. 
GTPases that exist in organisms such as C. elegans. These regulators likely provide the signaling specificity and spatial-temporal regulation required by the broadly expressed and functionally important Rho family GTPases. C. elegans unc-73 is interesting in this regard since it is the only gene, aside from its orthologs, that encodes two RhoGEF domains. We have previously shown that mutations that affect the $\mathrm{N}$-terminal region of UNC-73, which contains the Rac-selective RhoGEF-1 domain, interfere with guided cell movements and axonal growth cone migrations. Indeed, a substitution that specifically blocks RhoGEF-1 activity induces multiple axon guidance defects due to a failure to stimulate Rac family GTPases and downstream reorganization of the actin cytoskeleton. All such unc-73 mutations that yield an uncoordinated phenotype (Unc) cluster in the region encoding the unc-73 B transcript.

Our present analysis indicates that unc-73 encodes at least eight different transcripts as a consequence of alternative transcriptional start sites and $3^{\prime}$ ends. Six of the transcripts $(\mathrm{C} 1-\mathrm{F})$ derive from the $3^{\prime}$ half of the unc-73 gene and contain the RhoGEF-2 domain, which is specific for the Rho GTPase (Spencer et al. 2001). We have used reverse genetics and isoform-specific rescue experiments to examine the function of the UNC-73 RhoGEF-2 domain encoded by the C1, C2, D1, D2, E, and $\mathrm{F}$ transcripts, which are not perturbed in any of the Unc mutants.

We find that UNC-73 D1 and D2 function in pharyngeal muscle to regulate pharynx pumping and in the HSN neurons and vulval muscles to regulate egg laying, while UNC-73 C1, C2/F, and E function in the nervous system to regulate the rate of locomotion. Mutational analysis indicates all of these isoforms require a functional RhoGEF-2 domain, and interestingly, neurons appear more sensitive to the requirement for UNC-73 RhoGEF-2 activity. Previous work has suggested functions for the C. elegans Rho GTPase in cytokinesis and the migration of hypodermal cells (Jantsch-Plunger et al. 2000; Spencer et al. 2001). Our characterization of the UNC-73 C1-F isoforms suggests a role for the Rho signaling pathway in muscle cells and the neurons of adult animals. The C. elegans unc-73 gene is therefore a complex locus. It encodes multiple protein isoforms with different structures and distinct patterns of expression, and employs two RhoGEF domains with separate specificities to control multiple aspects of cellular organization and function during development and in the adult animal.

\section{The UNC-73/Trio/Kalirin family of RhoGEFs}

unc-73 belongs to a family that includes Drosophila (d) Trio and two separate mammalian genes, Trio and Kalirin. The Trio and Kalirin proteins have a similar domain organization to UNC-73, although each has a C-terminal serine/threonine kinase-like domain not present in unc73 and Trio does not have a FnIII domain. dTrio does not have the kinase, Ig or FnIII domains. The splicing of dTrio is quite similar to that of unc-73; the most notable exception being a lack of an equivalent UNC-73 B transcript (Awasaki et al. 2000; http://flybase.org). This recent discovery of the dTrio equivalents of the UNC-73 C2, D2, and E RhoGEF-2 transcripts may complicate previous conclusions regarding the function of the dTrio RhoGEF-2 domain since the dTrio mutations examined almost certainly do not affect these transcripts.

The splicing of Kalirin and Trio is somewhat similar to that of unc-73, but Kalirin has more alternative transcriptional start sites and $3^{\prime}$ ends and Trio encodes fewer transcripts (McPherson et al. 2002, 2004, 2005). An important difference in splicing from unc-73 involves the Kalirin 7 isoform, which is similar to UNC-73 B except that the SH3 domain is replaced with a short segment ending with a PDZ-binding motif (Penzes et al. 2003). We have not seen any evidence for a Kalirin 7-like isoform in our analysis of the unc-73 splice variations. In general, the expression of the Kalirin transcripts also appears similar to unc-73, while Trio expression is more widespread (Debant et al. 1996). Most of the UNC-73 and Kalirin isoforms are strongly expressed in the nervous system, except for UNC-73 D1 and D2 and the equivalent Kalirin "Duet" isoforms, which are more strongly expressed in muscle (Kawai et al. 1999; McPherson et al. 2002). The complexity of the alternative splicing and expression of unc-73 and its homologs points to the importance of RhoGEFs in controlling Rho family GTPases in multiple cell types and at different stages in development.

Several experiments indicate a role for Kalirin 7 at the mammalian synapse. Immunostaining and subcellular fractionation locates Kalirin 7 at the post-synaptic density, it interacts with several PDZ domain proteins found at the synapse and overexpression of Kalirin 7 increases; while reducing Kalirin 7 decreases, the number of dendritic spines in cultured hippocampal neurons ( $\mathrm{Ma}$ et al. 2003; Penzes et al. 2003). Importantly, although we believe that UNC-73 influences neurotransmission, $C$. elegans lacks dendritic spines, suggesting that Kalirin 7 may represent an evolutionary modification of the basic UNC-73 organization to accommodate more sophisticated neuronal functions.

\section{UNC-73 D1 and D2 have essential functions in pharynx muscle}

The deletion alleles ev802 and ev803, which lack the unc-73 RhoGEF-2 coding region, result in an L1 stage lethal phenotype not associated with any of the previously described unc-73 mutations. ev802 animals have a reduced rate and intensity of pharynx pumping, and they behave as if they were starved, arresting development at the L1 stage while remaining alive and coordinated in movement for several days. The ev802 L1 arrest is due to the lack of UNC-73 D1 and D2 in the muscles of the pharynx, since the developmental arrest is rescued with either D1 or D2 under the control of their endogenous promoters or with restricted expression of D1 in pharynx muscle cells alone. The general morphology of the pharynx in ev802 animals is normal, and no pharynx muscle 
abnormalities are detected under polarized light (data not shown); however, it is possible that the loss of D1 and D2 has a subtle affect on muscle structure. Although they are not found in D2, the Ig and FNIII regions of the D1 isoform are also suggestive of muscle-specific function. Combinations of these domains are most commonly associated with the extracellular regions of transmembrane receptor proteins, but they are also found in intracellular muscle proteins such as titin, myosin-binding protein $\mathrm{C}$, and myosin light chain kinase, which either play a structural role in setting up the muscle sarcomere or regulate muscle contraction (Benian et al. 1996).

Another possibility is that the D1 and D2 RhoGEF-2 domains may influence the activation state of Rho at pharynx neuromuscular junctions, allowing proper acetylcholine receptor clustering and efficient synaptic transmission (Weston et al. 2003). Cell ablation and pharynx dissection experiments, however, suggest that simple elimination of synaptic contacts with the pharyngeal or extrapharyngeal nervous systems would not explain the lethal ev802 phenotype (Avery and Horvitz 1989; Avery et al. 1995).

\section{The UNC-73 C1, C2/F, and E RhoGEF-2 isoforms regulate locomotion}

Although the UNC-73 D1 and D2 isoforms rescue ev802 lethality, the rescued animals have a unique lethargic movement phenotype. This movement phenotype is rescued, in turn, by any one of the UNC-73 C1, C2/F, or E isoforms. Several observations suggest that $\mathrm{C} 1, \mathrm{C} 2 / \mathrm{F}$, and $\mathrm{E}$ may function through their RhoGEF-2 domains to regulate neurotransmission. These include the findings that (1) the lethargy of [D1 or D2] ev802 animals can be overcome briefly by mechanical stimulation; (2) the expression of $\mathrm{C} 1, \mathrm{C} 2 / \mathrm{F}$, and $\mathrm{E}$ only coincides in the nervous system and [D1] ev802 animals do not have axon guidance defects; (3) the lethargic movement phenotype is related to that of $r b f-1$ rabphilin and unc-31 CAPS, which have roles in neurotransmission (Staunton et al. 2001; Speidel et al. 2005); and (4) the C1984Y and Q1944A substitutions in the RhoGEF-2 domain both abrogate rescue of the lethargic movement defect. Like $C$. elegans rbf-1 mutants, [D1] ev802 animals are not resistant to the acetylcholinesterase inhibitor aldicarb, which indicates UNC-73 is not important for acetylcholine synapse function and is thus unlikely to play a general role at all synapses. Consistent with this, synaptic structure was grossly normal in [D1] ev802 animals, as assayed using three different synaptic vesicle markers, indicating any defects at the synapses of these mutants do not affect synaptic vesicle patterning and/or the defects are specific to neurons not examined with these markers. The structure of the synapse also appears normal in $r b f-1$ and unc-31 mutants (Staunton et al. 2001; Cai et al. 2004).

Our genetic analysis indicates that [D1] ev802 lethargy is not a result of excessive serotonin or dopamine signaling, or due to an effect on SNARE protein function as reported for $r b f-1$ (Staunton et al. 2001). C. elegans locomotion is influenced by $G$ protein signaling through DGK (Nurrish et al. 1999) and Rho is an upstream inhibitor of DGK $\theta$ in mammalian cells (Houssa et al. 1999). DGK activity reduces the amount of diacylglycerol at the synaptic membrane, which has an inhibitory effect on neurotransmission. Based on the work of Houssa et al., we investigated whether a C. elegans DGK mutant might suppress [D1] ev802 lethargy. We instead found that UNC-73 C1, C2/F, and E act downstream of or parallel to DGK and $\mathrm{G}_{\mathrm{o}} \alpha$ signaling, which is in agreement with others who have shown that Rho family GTPases act downstream of DGK $\gamma$ and $\mathrm{G} \alpha$ proteins (Hart et al. 1998; Tsushima et al. 2004).

There are two general classes of vesicles found at the synapse: small, clear vesicles that contain neurotransmitters such as acetylcholine, and large, dense core vesicles (DCVs) that hold biogenic amines or neuropeptides. Neuropeptides such as the FMRF (Phe-Met-ArgPhe)amide-related group of neuromodulators are important signaling molecules in the nervous system, which modulate neuronal activity and influence many behaviors, including locomotion. An interesting possibility is that UNC-73 C1, C2/F, and E may regulate the secretion of neuropeptides or biogenic amines via DCVs. Although the FMRFamide level of [D1] ev802 animals appears normal (data not shown), analysis of mammalian rabphilin, CAPS/UNC-31, Kalirin, and Trio has suggested a role for these proteins in the process of regulated secretion via DCVs.

Rabphilin interacts preferentially with Rab27 (Fukuda et al. 2004), which is a regulator of exocytosis (Izumi et al. 2003), and rabphilin enhances DCV exocytosis in adrenal chromaffin cells (Chung et al. 1995). Mammalian CAPS/UNC-31 was proposed to function in calcium regulated DCV fusion, but recent analysis suggests that CAPS may instead act to load or maintain neurotransmitters in DCVs (Grishanin et al. 2004; Speidel et al. 2005). Kalirin and Trio also have connections to DCV secretion. Kalirin was initially identified through its interaction with a DCV-associated protein, and Kalirin enhances regulated ACTH secretion in anterior pituitary AtT-20 cells (Mains et al. 1999). Trio is implicated in hormone secretion downstream of the cdk5 kinase in primary cultures of anterior pituitary cells (Xin et al. 2004).

The fact that the phenotype of a [D1] unc-73(ev802); unc-31 double mutant is enhanced compared with the phenotypes of the single mutants suggests that unc-73 and unc-31 are not in the same linear pathway. However, the secretion of neurotransmitters from DCVs is a complex process, and considering the aforementioned functions of rabphilin, CAPS, and the UNC-73 orthologs, the lethargic movement phenotypes of C. elegans rbf-1, unc31 , and [D1] ev802 may reflect defects in separate aspects of the secretion process.

A role for UNC-73, Kalirin and Trio in DCV secretion could involve the regulation of actin. Yeast genetics and experiments using actin inhibitors on cultured endocrine cells and neurons indicate that actin is important 
for secretion and neurotransmitter release (Doussau and Augustine 2000; Sankaranarayanan et al. 2003; Bader et al. 2004). Also interesting, considering the importance of the UNC-73 RhoGEF-2 domain for locomotion, are the findings that Rho is associated with the membrane of DCVs in chromaffin cells, and Rho plays a role in exocytosis and endocytosis (Gasman et al. 1998; Ellis and Mellor 2000). We conclude that the UNC-73 C1, C2/F, and $\mathrm{E}$ isoforms, via their RhoGEF-2 domains, are required to activate Rho in the nervous system to modulate neurotransmission potentially through the control of DCV secretion.

\section{Materials and methods}

\section{C. elegans strains}

ev802 and ev803 were isolated from our frozen UV-TMP-induced C. elegans mutation library. bas-1(ad446) MT7988 and tph-1(mg280) are alleles that were used but not specified in the text. Double-mutant strains were constructed using standard genetic methods without additional marker mutations. The presence of the mutations was confirmed by PCR or outcrossing to him-5 males and examination of the F2 progeny.

\section{Molecular biology}

mRNA isolated from mixed stage N2 nematodes was used with SuperScript II reverse transcriptase (Invitrogen) and random hexamers or an oligo dT primer to generate cDNAs for use in RT-PCR. Each Northern blot lane contains $3 \mu \mathrm{g}$ of N2 mixed stage mRNA.

Nematode protein lysates were prepared by either sonication, for small samples, or three passages through an Emulsiflex tissue homogenizer for samples of $\geq 10 \mathrm{~mL}$. Immunoprecipitations used $1 \mathrm{mg}$ of soluble protein lysate, $5 \mu \mathrm{g}$ of antibody, and $10 \mu \mathrm{L}$ of protein A-Sepharose.

\section{unc-73 constructs}

All PCR-generated fragments used for cloning were sequenced in their entirety, and the cloning junctions in the final constructs were also sequenced. SunI-digested C11B5 cosmid was religated to make $\Delta \mathrm{C} 11 \mathrm{~B} 5$. The construct $\mathrm{pNW} 217$, which encodes only the UNC-73 B isoform, was previously described (Supplementary Fig. S2; Steven et al. 1998). A filled-in Asp718I digest and subsequent SmaI digest of pPD95.02 yielded GFP, which was cloned into a unique blunted ClaI site in pNW217 placing GFP in frame between the $\mathrm{PH}$ and $\mathrm{SH} 3$ domains of UNC-73 B. This construct, named pNW248, rescued the unc73(gm40) Unc and Let phenotypes. The UNC-73 E specific construct is a 10-kb BclI genomic fragment from C11B5 (Fig. 1; Supplementary Fig. S2) subcloned into the BamHI site of pBluescript. A rescue capable GFP-tagged version of this construct was made by cloning a blunted KpnI BstXI pPD95.02 fragment into a blunted PflMI site located at the very 3 ' end of the coding region.

UNC-73 C1, C2/F, D1, and D2 constructs were each made with three PCR-generated DNA fragments with convenient restriction enzyme sites introduced at their ends (Supplementary Fig. S2). The most 5 ' fragment was made using a genomic DNA template (C11B5) and included all of the intron sequence (presumed to be promoter) (Fig. 2) immediately upstream of the transcript's first exon, the first exon, and the first intron. The middle cDNA fragment encodes from the second exon to the last exon. The last genomic fragment extended $2 \mathrm{~kb}$ from the last exon, for the $\mathrm{C} 1$ and D1 constructs, or the full length of intron 26, for the C2/F and D2 constructs. The three fragments were cloned into pBluescript. Each construct encodes an individual unc-73 transcript except for $\mathrm{C} 2 / \mathrm{F}$, which encodes both the $\mathrm{C} 2$ and $\mathrm{F}$ transcripts.

DNA sequence changes made to facilitate cloning resulted in minor alterations to the protein sequences (Supplemental Material). PCR-based site-directed mutagenesis (Expand Long Template Polymerase; Roche Applied Science) was used to introduce the Q1944A and C1984Y mutations.

GFP was introduced into the $\mathrm{C} 1$ and $\mathrm{D} 1$ constructs by cloning in a blunted KpnI BstXI pPD95.02 fragment into a unique StuI site generated at the $3^{\prime}$ end of the coding sequence (Supplementary Fig. S2). A partially blunted Asp718I SmaI pPD95.02 fragment was cloned into similar StuI sites in the C2 and D2 constructs. All GFP-tagged isoforms behaved the same as the corresponding nontagged versions in rescue experiments except for $\mathrm{C} 1:: \mathrm{GFP}$ and $\mathrm{C} 2:: \mathrm{GFP}$, which did not rescue the lethargy of [D1] ev802 animals.

myo-2p::D1 and myo-2p::D1::GFP plasmids were generated by replacing the ApaI Asp718I promoter-containing fragments from the D1 and D1 ::GFP constructs with an XbaI NheI myo-2 promoter fragment from pPD132.102.

\section{Transgenic lines}

Standard microinjection techniques were used to generate stable transgenic C. elegans lines carrying extrachromosomal DNA arrays. Between 20 and $50 \mathrm{ng} / \mu \mathrm{L}$ of DNA per construct was injected. F25B3.3p::GFP (pDP\#SU006), sur-5::GFP or pRF4 [rol-6(d)] were used as cotransformation markers. At least three lines were isolated for each set of plasmids that were injected, and each set of lines had the same phenotype or identical GFP expression pattern. The arrays in these lines were not integrated into the genome, although the array in line [D1; F25B3.3p::GFP] ev802-A spontaneously integrated into LG V.

F25B3.3p::GFP, which is expressed in the nervous system, was the cotransformation marker used for most rescue experiments. However, there was an inconsistency specific to [D2; F25B3.3p::GFP] ev802 lines whereby the animals originally appeared lethargic, but after several generations, they moved at rates closer to wild type. A possible explanation is that recombination occurred between F25B3.3p:: GFP and the D2 plasmid so D2 became expressed in the nervous system, which corrected the lethargy. Consistent with this explanation, [D2; pRF4] ev802 lines were stable for the lethargic phenotype. This phenomenon did not occur with [D1; F25B3.3p::GFP] ev802 or [myo-2p::D1; F25B3.3p::GFP] ev802 lines, which have always remained lethargic.

To test their ability to rescue ev802 lethality, the isoformspecific constructs were injected into ev802/unc-11dpy-5 animals. Usually, while screening F2 animals for stable lines, we immediately found ev802/ev802 lines if that particular construct was capable of rescuing ev802 lethality. All of the adult F2 animals of a rescuing ev802/ev802 line fluoresced due to the cotransformation marker, and no DpyUnc progeny were present. If ev802/ev802 lines were not immediately obtained, adults from established ev802/unc-11dpy-5 transgenic lines containing potential rescuing arrays were left to lay eggs for 24 $\mathrm{h}$, and at least 40 transgenic nonDpy larval animals per line were cloned the next day. If all clones segregated DpyUnc progeny, we concluded the injected construct(s) did not rescue ev802 lethality. 


\section{Phenotypic analysis}

Pharynx pumping rates were measured using digital video of either healthy young adults or L1 animals moving freely on a bacterial lawn. At least 10 animals per genotype were examined with two 20 -sec periods of consistent pharynx pumping observed per animal.

Body bends were counted while healthy young adults moved uninterrupted at room temperature on plates without bacteria. At least 10 animals per genotype were examined with three 20 -sec periods of movement observed per animal. Animals on plates with bacteria were transferred to plates without bacteria and left for 2 min before counting. If an animal stopped moving or reversed direction, that count was abandoned. One body bend is defined as the motion of the terminal bulb starting from the top position of the sinusoidal wave track through to the bottom and back to the top.

Strains were examined for egg laying defects by starting with mid L4 animals and checking for live larvae within each animal daily for a total of 3 consecutive days. Animals were considered Egl if they held at least one live larvae by the end of the 3-d period, although most Egl animals had a "bag of worms" phenotype before the end of $3 \mathrm{~d}$. Rare sterile animals were eliminated from the totals.

\section{Microscopy}

Pharynx pumping was captured on digital video with a Nikon Coolpix 4500 camera mounted to a Zeiss Stemi SV 11 microscope. Fluorescent protein expression in live (immobilized with $10 \mathrm{mM}$ levamisole in $\mathrm{M} 9$ ) worms was viewed with a Leica DMRA2 microscope and images recorded with a Hamamatsu ORCA-ER camera and Openlab software (Improvision).

\section{Acknowledgments}

We thank Theresa Steirnagle at the Caenorhabditis Genetics Center (which is funded by the National Institutes of Health [NIH] National Center for Research Resources), Mike Nonet, Bill Nuttley, and Jim Rand for nematode strains; Yuji Kohara for cDNAs; Andy Fire and Dave Pilgrim for plasmids; Mei Zhen for juIs1; Martin Chalfie for sek-1::snb-1::gfp; John Hutton for ida$1:: g f p$; Hubert van Tol and Sandi Ordog for movement measurements; Jim McGhee and Jay Kormish for fluorescent beads; Stephan Black for technical assistance; Chris Li for the antiRFamide antibody; and Terry Kubiseski for critical reading of the manuscript. This work was supported by grant FRN 36651 from the Canadian Institutes for Health Research (CIHR) and grant \#5 R01 NS 041397 from NIH, and post-doctoral fellowships to R.S. from the PVA Spinal Cord Research Foundation and the CIHR Canadian Neurotrauma Research Program. T.P. is a distinguished scientist of the CIHR.

\section{References}

Alam, M.R., Johnson, R.C., Darlington, D.N., Hand, T.A., Mains, R.E., and Eipper, B.A. 1997. Kalirin, a cytosolic protein with spectrin-like and GDP/GTP exchange factor-like domains that interacts with peptidylglycine $\alpha$-amidating monooxygenase, an integral membrane peptide-processing enzyme. J. Biol. Chem. 272: 12667-12675.

Avery, L. and Horvitz, H.R. 1989. Pharyngeal pumping continues after laser killing of the pharyngeal nervous system of $C$. elegans. Neuron 3: 473-485.

Avery, L., Bargmann, C.I., and Horvitz, H.R. 1993. The Cae- norhabditis elegans unc-31 gene affects multiple nervous system-controlled functions. Genetics 134: 455-464.

Avery, L., Raizen, D.M., and Lockery, S. 1995. Electrophysiological methods. In Methods in cell biology: C elegans: Modern biological analysis of an organism (eds. H.F. Epstein and D.C. Shakes), pp. 251-269. Academic Press, San Diego.

Awasaki, T., Saito, M., Sone, M., Suzuki, E., Sakai, R., Ito, K., and Hama, C. 2000. The Drosophila trio plays an essential role in patterning of axons by regulating their directional extension. Neuron 26: 119-131.

Bader, M.F., Doussau, F., Chasserot-Golaz, S., Vitale, N., and Gasman, S. 2004. Coupling actin and membrane dynamics during calcium-regulated exocytosis: A role for Rho and ARF GTPases. Biochim. Biophys. Acta 1742: 37-49.

Bateman, J., Shu, H., and Van Vactor, D. 2000. The guanine nucleotide exchange factor trio mediates axonal development in the Drosophila embryo. Neuron 26: 93-106.

Benian, G.M., Tang, X., and Tinley, T.L. 1996. Twitchin and related giant Ig superfamily members of $C$. elegans and other invertebrates. Adv. Biophys. 33: 183-198.

Burridge, K. and Wennerberg, K. 2004. Rho and Rac take center stage. Cell 116: 167-179.

Cai, T., Fukushige, T., Notkins, A.L., and Krause, M. 2004. Insulinoma-associated protein IA-2, a vesicle transmembrane protein, genetically interacts with UNC-31/CAPS and affects neurosecretion in Caenorhabditis elegans. J. Neurosci. 24: 3115-3124.

Chalfie, M. and Sulston, J. 1981. Developmental genetics of the mechanosensory neurons of Caenorhabditis elegans. Dev. Biol. 82: 358-370.

Chung, S.H., Takai, Y., and Holz, R.W. 1995. Evidence that the Rab3a-binding protein, rabphilin3a, enhances regulated secretion: Studies in adrenal chromaffin cells. J. Biol. Chem. 270: 16714-16718.

Debant, A., Serra-Pages, C., Seipel, K., O'Brien, S., Tang, M., Park, S.H., and Streuli, M. 1996. The multidomain protein Trio binds the LAR transmembrane tyrosine phosphatase, contains a protein kinase domain, and has separate rac-specific and rho-specific guanine nucleotide exchange factor domains. Proc. Nat1. Acad. Sci. 93: 5466-5471.

Doussau, F. and Augustine, G.J. 2000. The actin cytoskeleton and neurotransmitter release: An overview. Biochimie 82: 353-363.

Ellis, S. and Mellor, H. 2000. Regulation of endocytic traffic by rho family GTPases. Trends Cell Biol. 10: 85-88.

Fukuda, M., Kanno, E., and Yamamoto, A. 2004. Rabphilin and Noc2 are recruited to dense-core vesicles through specific interaction with Rab27A in PC12 cells. J. Biol. Chem. 279: 13065-13075.

Gasman, S., Chasserot-Golaz, S., Hubert, P., Aunis, D., and Bader, M.F. 1998. Identification of a potential effector pathway for the trimeric Go protein associated with secretory granules: Go stimulates a granule-bound phosphatidylinositol 4-kinase by activating RhoA in chromaffin cells. J. Biol. Chem. 273: 16913-16920.

Grishanin, R.N., Kowalchyk, J.A., Klenchin, V.A., Ann, K., Earles, C.A., Chapman, E.R., Gerona, R.R., and Martin, T.F. 2004. CAPS acts at a prefusion step in dense-core vesicle exocytosis as a PIP2 binding protein. Neuron 43: 551-562.

Hallam, S.J. and Jin, Y. 1998. lin-14 regulates the timing of synaptic remodelling in Caenorhabditis elegans. Nature 395: 78-82.

Hart, M.J., Jiang, X., Kozasa, T., Roscoe, W., Singer, W.D., Gilman, A.G., Sternweis, P.C., and Bollag, G. 1998. Direct stimulation of the guanine nucleotide exchange activity of p115 RhoGEF by Ga13. Science 280: 2112-2114. 
Horvitz, H.R., Chalfie, M., Trent, C., Sulston, J.E., and Evans, P.D. 1982. Serotonin and octopamine in the nematode Caenorhabditis elegans. Science 216: 1012-1014.

Houssa, B., de Widt, J., Kranenburg, O., Moolenaar, W.H., and van Blitterswijk, W.J. 1999. Diacylglycerol kinase $\theta$ binds to and is negatively regulated by active RhoA. J. Biol. Chem. 274: 6820-6822.

Izumi, T., Gomi, H., Kasai, K., Mizutani, S., and Torii, S. 2003. The roles of Rab27 and its effectors in the regulated secretory pathways. Cell Struct. Funct. 28: 465-474.

Jantsch-Plunger, V., Gonczy, P., Romano, A., Schnabel, H., Hamill, D., Schnabel, R., Hyman, A.A., and Glotzer, M. 2000. CYK-4: A Rho family GTPase activating protein (GAP) required for central spindle formation and cytokinesis. I. Cell. Biol. 149: 1391-1404.

Kawai, T., Sanjo, H., and Akira, S. 1999. Duet is a novel serine/ threonine kinase with Dbl-Homology (DH) and PleckstrinHomology (PH) domains. Gene 227 249-255.

Kubiseski, T.J., Culotti, J., and Pawson, T. 2003. Functional analysis of the Caenorhabditis elegans UNC-73B PH domain demonstrates a role in activation of the Rac GTPase in vitro and axon guidance in vivo. Mol. Cell. Biol. 23: 68236835.

Liebl, E.C., Forsthoefel, D.J., Franco, L.S., Sample, S.H., Hess, J.E., Cowger, J.A., Chandler, M.P., Shupert, A.M., and Seeger, M.A. 2000. Dosage-sensitive, reciprocal genetic interactions between the Abl tyrosine kinase and the putative GEF trio reveal trio's role in axon pathfinding. Neuron 26: 107-118.

Liu, X., Wang, H., Eberstadt, M., Schnuchel, A., Olejniczak, E.T., Meadows, R.P., Schkeryantz, J.M., Janowick, D.A., Harlan, J.E., Harris, E.A., et al. 1998. NMR structure and mutagenesis of the $\mathrm{N}$-terminal Dbl homology domain of the nucleotide exchange factor Trio. Cell 95: 269-277.

Loer, C.M. and Kenyon, C.J. 1993. Serotonin-deficient mutants and male mating behavior in the nematode Caenorhabditis elegans. J. Neurosci. 13: 5407-5417.

Lundquist, E.A., Reddien, P.W., Hartwieg, E., Horvitz, H.R., and Bargmann, C.I. 2001. Three C. elegans Rac proteins and several alternative Rac regulators control axon guidance, cell migration and apoptotic cell phagocytosis. Development 128: $4475-4488$.

Ma, X.M., Huang, J., Wang, Y., Eipper, B.A., and Mains, R.E. 2003. Kalirin, a multifunctional Rho guanine nucleotide exchange factor, is necessary for maintenance of hippocampal pyramidal neuron dendrites and dendritic spines. J. Neurosci. 23: 10593-10603.

Mains, R.E., Alam, M.R., Johnson, R.C., Darlington, D.N., Back, N., Hand, T.A., and Eipper, B.A. 1999. Kalirin, a multifunctional PAM COOH-terminal domain interactor protein, affects cytoskeletal organization and ACTH secretion from AtT-20 cells. J. Biol. Chem. 274: 2929-2937.

McPherson, C.E., Eipper, B.A., and Mains, R.E. 2002. Genomic organization and differential expression of Kalirin isoforms. Gene 284: 41-51.

- 2004. Kalirin expression is regulated by multiple promoters. J. Mol. Neurosci. 22: 51-62.

- 2005. Multiple novel isoforms of Trio are expressed in the developing rat brain. Gene 347: 125-135.

Mendel, J.E., Korswagen, H.C., Liu, K.S., Hajdu-Cronin, Y.M., Simon, M.I., Plasterk, R.H., and Sternberg, P.W. 1995. Participation of the protein Go in multiple aspects of behavior in C. elegans. Science 267: 1652-1655.

Miller, K.G., Alfonso, A., Nguyen, M., Crowell, J.A., Johnson, C.D., and Rand, J.B. 1996. A genetic selection for Caenorhabditis elegans synaptic transmission mutants. Proc. Natl. Acad. Sci. 93: 12593-12598.
Moon, S.Y. and Zheng, Y. 2003. Rho GTPase-activating proteins in cell regulation. Trends Cell Biol. 13: 13-22.

Newsome, T.P., Schmidt, S., Dietzl, G., Keleman, K., Asling, B., Debant, A., and Dickson, B.J. 2000. Trio combines with dock to regulate Pak activity during photoreceptor axon pathfinding in Drosophila. Cell 101: 283-294.

$\mathrm{Ng}$, J. and Luo, L. 2004. Rho GTPases regulate axon growth through convergent and divergent signaling pathways. Neuron 44: 779-793.

Nonet, M.L., Holgado, A.M., Brewer, F., Serpe, C.J., Norbeck, B.A., Holleran, J., Wei, L., Hartwieg, E., Jorgensen, E.M., and Alfonso, A. 1999. UNC-11, a Caenorhabditis elegans AP180 homologue, regulates the size and protein composition of synaptic vesicles. Mol. Biol. Cell 10: 2343-2360.

Nurrish, S., Segalat, L., and Kaplan, J.M. 1999. Serotonin inhibition of synaptic transmission: $\mathrm{G} \alpha(0)$ decreases the abundance of UNC-13 at release sites. Neuron 24: 231-242.

Olofsson, B. 1999. Rho guanine dissociation inhibitors: Pivotal molecules in cellular signalling. Cell Signal 11: 545-554.

Penzes, P., Beeser, A., Chernoff, J., Schiller, M.R., Eipper, B.A., Mains, R.E., and Huganir, R.L. 2003. Rapid induction of dendritic spine morphogenesis by trans-synaptic ephrinB-EphB receptor activation of the Rho-GEF kalirin. Neuron 37: 263274.

Rossman, K.L., Der, C.J., and Sondek, J. 2005. GEF means go: Turning on RHO GTPases with guanine nucleotide-exchange factors. Nat. Rev. Mol. Cell. Biol. 6: 167-180.

Sankaranarayanan, S., Atluri, P.P., and Ryan, T.A. 2003. Actin has a molecular scaffolding, not propulsive, role in presynaptic function. Nat. Neurosci. 6: 127-135.

Sawin, E.R., Ranganathan, R., and Horvitz, H.R. 2000. C. elegans locomotory rate is modulated by the environment through a dopaminergic pathway and by experience through a serotonergic pathway. Neuron 26: 619-631.

Schafer, W.R. and Kenyon, C.J. 1995. A calcium-channel homologue required for adaptation to dopamine and serotonin in Caenorhabditis elegans. Nature 375: 73-78.

Segalat, L., Elkes, D.A., and Kaplan, J.M. 1995. Modulation of serotonin-controlled behaviors by Go in Caenorhabditis elegans. Science 267: 1648-1651.

Speidel, D., Bruederle, C.E., Enk, C., Voets, T., Varoqueaux, F., Reim, K., Becherer, U., Fornai, F., Ruggieri, S., Holighaus, Y., et al. 2005. CAPS1 regulates catecholamine loading of large dense-core vesicles. Neuron 46: 75-88.

Spencer, A.G., Orita, S., Malone, C.J., and Han, M. 2001. A RHO GTPase-mediated pathway is required during $\mathrm{P}$ cell migration in Caenorhabditis elegans. Proc. Natl. Acad. Sci. 98 13132-13137.

Staunton, J., Ganetzky, B., and Nonet, M.L. 2001. Rabphilin potentiates soluble $\mathrm{N}$-ethylmaleimide sensitive factor attachment protein receptor function independently of rab3. J. Neurosci. 21 9255-9264.

Steven, R., Kubiseski, T.J., Zheng, H., Kulkarni, S., Mancillas, J., Ruiz Morales, A., Hogue, C.W., Pawson, T., and Culotti, J. 1998. UNC-73 activates the Rac GTPase and is required for cell and growth cone migrations in C. elegans. Cell 92 785795.

Sze, J.Y., Victor, M., Loer, C., Shi, Y., and Ruvkun, G. 2000. Food and metabolic signalling defects in a Caenorhabditis elegans serotonin-synthesis mutant. Nature 403: 560-564.

Trent, C., Tsuing, N., and Horvitz, H.R. 1983. Egg-laying defective mutants of the nematode Caenorhabditis elegans. Genetics 104: 619-647.

Tsushima, S., Kai, M., Yamada, K., Imai, S., Houkin, K., Kanoh, H., and Sakane, F. 2004. Diacylglycerol kinase $\gamma$ serves as an upstream suppressor of Racl and lamellipodium formation. 
J. Biol. Chem. 279: 28603-28613.

Weston, C., Gordon, C., Teressa, G., Hod, E., Ren, X.D., and Prives, J. 2003. Cooperative regulation by Rac and Rho of agrin-induced acetylcholine receptor clustering in muscle cells. J. Biol. Chem. 278: 6450-6455.

Wu, Y.C., Cheng, T.W., Lee, M.C., and Weng, N.Y. 2002. Distinct rac activation pathways control Caenorhabditis elegans cell migration and axon outgrowth. Dev. Biol. 250: $145-155$.

Xin, X., Ferraro, F., Back, N., Eipper, B.A., and Mains, R.E. 2004. Cdk5 and Trio modulate endocrine cell exocytosis. J. Cell. Sci. 117: 4739-4748.

Zahn, T.R., Macmorris, M.A., Dong, W., Day, R., and Hutton, J.C. 2001. IDA-1, a Caenorhabditis elegans homolog of the diabetic autoantigens IA-2 and phogrin, is expressed in peptidergic neurons in the worm. I. Comp. Neurol. 429: 127143. 


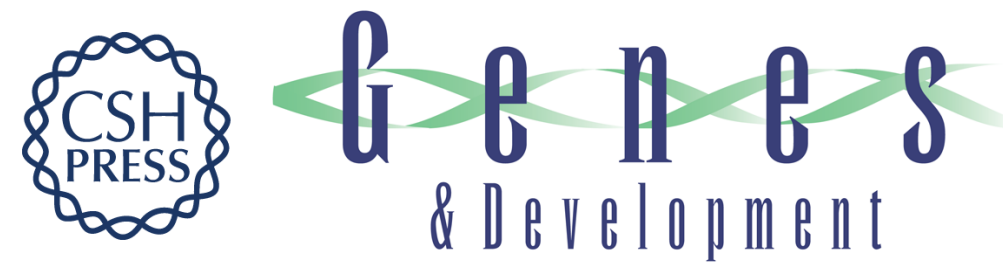

\section{The UNC-73/Trio RhoGEF-2 domain is required in separate isoforms for the regulation of pharynx pumping and normal neurotransmission in C. elegans}

Robert Steven, Lijia Zhang, Joseph Culotti, et al.

Genes Dev. 2005, 19:

Access the most recent version at doi:10.1101/gad.1319905

Supplemental http://genesdev.cshlp.org/content/suppl/2005/08/18/19.17.2016.DC1

Material

References This article cites 58 articles, 25 of which can be accessed free at:

http://genesdev.cshlp.org/content/19/17/2016.full.html\#ref-list-1

License

Email Alerting Receive free email alerts when new articles cite this article - sign up in the box at the top

Service right corner of the article or click here.

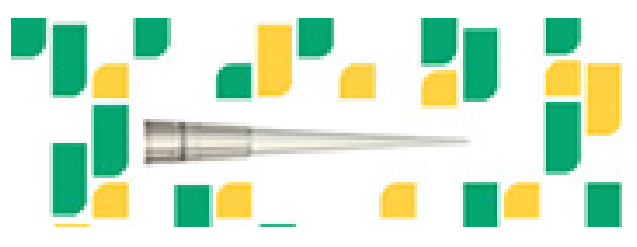

Focused on your science. 